\title{
Instrumentação virtual aplicada no desenvolvimento de uma bancada de ensaio de
}

\section{transformadores}

\author{
Virtual instrumentation applied in the development of a transformer test bench \\ Instrumentación virtual aplicada en el desarrollo de un banco de pruebas de transformadores
}

Recebido: 25/11/2021 | Revisado: 01/12/2021 | Aceito: 02/12/2021 | Publicado: 12/12/2021

\author{
Victor Henrique da Cunha Faria \\ ORCID: https://orcid.org/0000-0003-0063-6319 \\ Universidade Federal de Uberlândia, Brasil \\ E-mail: victorhcf@ufu.br \\ Leonardo Correa Caixeta \\ ORCID: https://orcid.org/0000-0001-5664-4102 \\ Centro Universitário de Patos de Minas, Brasil \\ E-mail: leoccaixeta@gmail.com \\ Gaspar Eugênio Oliveira Ramos \\ ORCID: https://orcid.org/0000-0002-0612-1343 \\ Centro Universitário de Patos de Minas, Brasil \\ E-mail: gasparramos@unipam.edu.br \\ Luciano Coutinho Gomes \\ ORCID: https://orcid.org/0000-0002-2827-6944 \\ Universidade Federal de Uberlândia, Brasil \\ E-mail:1cgomes@ufu.br
}

\begin{abstract}
Resumo
O transformador é importante em diversas áreas da engenharia elétrica e em busca de verificar a eficiência desses equipamentos são realizados procedimentos conhecidos como ensaios. Os ensaios de curto-circuito e circuito aberto são importantes por permitirem determinar grandezas que compõem o modelo real dos transformadores. O objetivo desse trabalho foi desenvolver um sistema automatizado de ensaios de curto-circuito e circuito aberto para transformadores monofásicos de baixa tensão utilizando instrumentação virtual através do LabVIEW, visando diminuir a interferência do operador e consequentemente, obter resultados mais precisos, em menos tempo e maior confiança. Para isso, realizou-se o estudo de bibliografias, a determinação dos sensores de corrente e tensão, a construção da bancada de ensaio, criação do software em LabVIEW, calibração, teste, aplicação, análise e apresentação dos resultados. O sistema desenvolvido apresentou resultados confiáveis, com baixo erro de medição, o que permitiu ao operador, uma vez montado o arranjo dos ensaios, a não necessidade de manipulação dos instrumentos convencionais durante os ensaios. Palavras-chave: Ensaio; Transformador; Automatizado; LabVIEW.
\end{abstract}

\begin{abstract}
The transformer is important in several areas of electrical engineering, so in order to verify the efficiency of the equipment, tests are performed. Short-circuit and open-circuit tests are relevant because they allow the determination of quantities that represents the real transformers model. The objective of this work was to develop an automated shortcircuit and open circuit testing system for low voltage single-phase transformers using virtual instrumentation through LabVIEW, reducing operator interference and, consequently, obtaining more accurate results, in less time and with greater confidence. Therefore, a bibliography study, the determination of current and voltage sensors, the construction of the test bench, creation of the software in LabVIEW, calibration, testing, application, analysis and presentation of results were carried out. The system presented accurate results, with low quality error, which allowed the operator, once the arrangement of tests has been assembled, the unnecessary use of instruments during the tests.
\end{abstract}

Keywords: Test; Transformer; Automated; LabVIEW.

\section{Resumen}

El transformador es importante en varias áreas de la ingeniería eléctrica y para verificar la eficiencia de este equipo se realizan procedimientos conocidos como pruebas. Las pruebas de cortocircuito y circuito abierto son importantes porque permiten determinar las magnitudes que componen el modelo real de los transformadores. El objetivo de este trabajo fue desarrollar un sistema automatizado de pruebas de cortocircuito y circuito abierto para transformadores monofásicos de baja tensión utilizando la instrumentación virtual LabVIEW, con el fin de reducir la interferencia del operador y, en consecuencia, obtener resultados más precisos en menos tiempo y con mayor confianza. Para ello, se realizó el estudio 
de bibliografía, la determinación de los sensores de corriente y tensión, la construcción del banco de pruebas, la creación del software en LabVIEW, la calibración, la prueba, la aplicación, el análisis y la presentación de los resultados. El sistema desarrollado presentaba resultados fiables, con un bajo error de medición, lo que permitía al operador, una vez montado el montaje de la prueba, no tener que manipular los instrumentos convencionales durante las pruebas.

Palabras clave: Prueba; Transformador; Automatizado; LabVIEW.

\section{Introdução}

A dependência humana da energia elétrica está cada vez maior em todas as áreas, sendo imprescindível para o pleno funcionamento da sociedade. Assim, uma pausa no fornecimento de eletricidade, mesmo que temporária, pode acarretar grandes prejuízos. Focando atender todo o consumo requerido, torna-se necessário o desenvolvimento de sistemas de geração, transmissão e distribuição de energia elétrica cada vez maiores, complexos, tecnológicos e eficientes para que não haja falhas no fornecimento (Araujo, 2009).

Os transformadores estão entre os principais e mais importantes equipamentos presentes no sistema elétrico de potência, os quais fazem parte dos sistemas de transmissão e distribuição (Barman, 2013). Visando garantir e atestar a eficiência desses equipamentos, ensaios de curto-circuito e de circuito aberto são realizados seguindo a norma ABNT NBR 5356-1:2007, em vista da determinação dos parâmetros elétricos do transformador, tais como: resistências, reatâncias, corrente de excitação, perdas no núcleo e perdas nos enrolamentos (Batista et al., 2010).

Os ensaios realizados manualmente podem ser fontes de erros nas medições e demandar um longo tempo de execução, levando em consideração que são usados instrumentos de medição convencionais (voltímetros, amperímetros e wattímetros), em que são operados manualmente. Ademais, durante todo o processo, o operador necessita fazer anotações dos valores exibidos pelos instrumentos (Batista et al., 2010).

Desse modo, tendo que os ensaios são feitos seguindo sempre os mesmos procedimentos (Bandeira, 2004), é possível o desenvolvimento de metodologias que eficientizem os ensaios, agregando maior velocidade e fidelidade na execução de cada teste, tornando-os automatizados. Isto, dispondo de sistemas de controle e aquisição de dados que minimizem o contato humano com os equipamentos de medição durante os ensaios, reduzindo os erros humanos nas medições e nos cálculos (Vicente, 2006).

Esses sistemas podem ser implementados utilizando instrumentos virtuais para agrupar todas as medições em um único dispositivo e as realizarem de forma automática, acrescentando confiabilidade metrológica aos processos de medição. Além disso, quando comparado a um instrumento de uso específico, a virtualização garante menor custo de aquisição e maior flexibilidade em fazer alterações. O ambiente de programação LabVIEW torna possível a implementação de um instrumento virtual. (Almeida et al., 2010).

Um instrumento virtual é integrado por ferramentas de programação e equipamentos de aquisição de sinais, que juntos a um computador, executam as mesmas funções de instrumentos convencionais (multímetros, osciloscópios, analisadores de energia, entre outros) (Cao \& Chen, 2014). Ultimamente, vários instrumentos, tanto analógicos quanto digitais, podem ser agrupados em um único instrumento virtual, permitindo que o espaço ocupado por um dispositivo convencional e os custos em sua produção sejam reduzidos. Dessa forma, em um único software, é viável mesclar várias funcionalidades de dispositivos convencionais (Kang et al., 2014).

Diante do exposto e visando maior praticidade na execução de ensaios em transformadores, o presente trabalho tem como objetivo o desenvolvimento de um sistema automatizado de ensaios de curto-circuito e ensaios de circuito aberto para transformadores monofásicos de baixa tensão utilizando a programação gráfica LabVIEW. 


\section{Metodologia}

\subsection{Delineamento experimental}

O referente trabalho teve como finalidade o desenvolvimento e implementação de um sistema automatizado de ensaios de curto-circuito e circuito aberto em transformadores monofásicos de baixa tensão com o emprego de medições e controles implementados, por meio de um instrumento virtual implementado com a linguagem de programação gráfica LabVIEW. Para tal, os métodos de pesquisa adotados neste projeto enquadram-se em bibliográfica, de campo exploratória, de natureza qualitativa e quantitativa.

A compreensão do modelo real para representar transformadores, tanto quanto os procedimentos convencionais de ensaios de transformadores, é primordial para o desenvolvimento da bancada automatizada de ensaios. Tais conhecimentos permitem sistematizar os processos a serem realizados pela bancada, buscando maior eficiência e velocidade nos ensaios de curto-circuito e circuito aberto.

\subsection{Modelo real do transformador}

O modelo real deseja exprimir com maior exatidão o comportamento de um transformador, levando em consideração as perdas que ocorrem na conversão de energia (Maia, 2013). De acordo com Baggini (2016), as perdas em transformadores podem ser divididas em dois principais grupos, as perdas sem carga e as perdas com carga. Essas perdas são comuns a todos os tipos de transformadores, independentemente da aplicabilidade e do nível de tensão. As perdas sem carga comtemplam perdas por histerese e perdas por correntes parasitas no núcleo, enquanto as perdas com carga englobam as perdas por aquecimento dos enrolamentos, conhecidas também como perdas no cobre.

As perdas por histerese estão relacionadas com a troca de polaridade dos domínios magnéticos, já que eles devem superar o atrito e a inércia para se orientarem no sentido do campo magnético aplicado, consumindo energia no processo. Enquanto que as perdas por corrente parasita no núcleo acontecem pelo surgimento de correntes elétricas induzidas no material, as quais surgem devido à variação do fluxo magnético no material, o que provoca a formação de um campo magnético de sentido contrário ao campo externo aplicado (Maria, 2015).

As perdas no cobre ocorrem pelo aquecimento gerado nos condutores presentes nos enrolamentos dos transformadores com a passagem de corrente elétrica, sendo então, perdas ocasionadas pelo efeito Joule (Prado, 2012). Com isso, é necessário construir um circuito equivalente que leve em consideração todas as mais significantes deficiências na transferência de energia dos transformadores reais.

Através de técnicas de representação de circuitos eletromagnéticos em circuitos elétricos, mostrada na Figura 1, retrata-se as grandezas reais em forma de impedância, mais especificamente como resistências e indutores, que por sua vez, caracterizam as principais perdas, perdas no cobre e perdas no núcleo (Toro, 2016). 
Figura 1: Circuito equivalente do modelo real de um transformador.

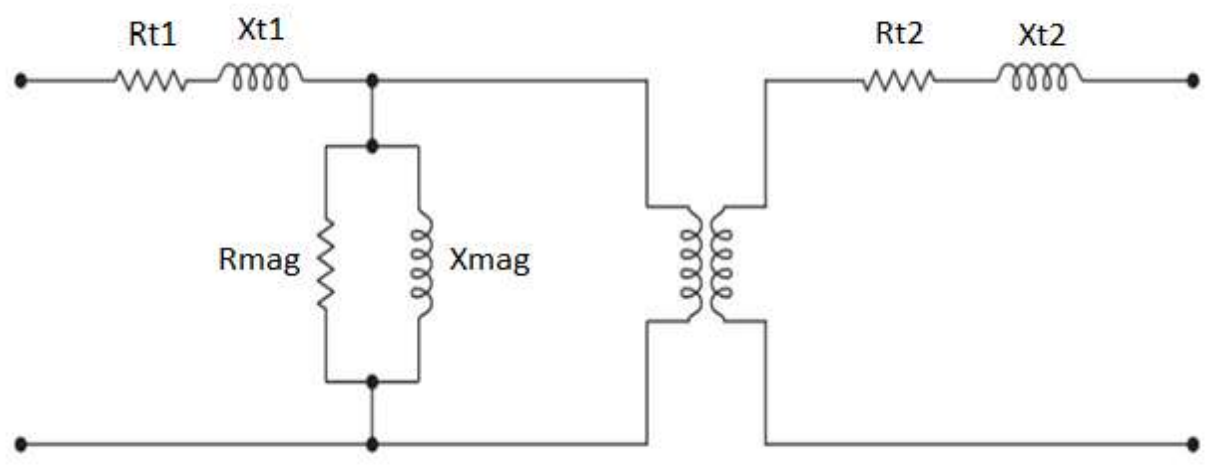

Fonte: Autores.

É possível observar ainda na Figura 1, que Rt1 e Rt2 são resistências em série que ilustram as perdas no cobre, que ocorrem devido ao efeito Joule nos enrolamentos. As grandezas Xt1 e Xt2 correspondem à reatância de dispersão que foi dividida entre o enrolamento primário e secundário. E no ramo paralelo, Rmag e Xmag, descrevem o comportamento do núcleo, abarcando as não linearidades da magnetização e o fenômeno das correntes parasitas. (Marín, 2016).

A determinação das referidas grandezas do circuito equivalente, resistências e reatâncias, podem ser obtidas experimentalmente através da realização de ensaios de curto-circuito e ensaios de circuito aberto (Chapman, 2013).

\subsection{Ensaio de curto-circuito e circuito aberto}

Ensaios são uma forma experimental de determinar as propriedades de um transformador. Os ensaios de curtocircuito e de circuito aberto têm a função de determinar os parâmetros do circuito equivalente do transformador (Umans, 2014).

$\mathrm{O}$ ensaio de circuito aberto consiste em fornecer tensão nominal ao transformador em um dos seus enrolamentos, deixando o outro enrolamento a vazio. Com isso, a passagem de corrente em suas bobinas poderá ser desconsiderada, obtendo somente as perdas no núcleo. Assim, o objetivo do ensaio de circuito aberto (Figura 2) é determinar as perdas no núcleo ferromagnético, a corrente a vazio e a impedância do ramo magnetizante. Recomenda-se que a conexão dos medidores e da fonte seja feita no lado de baixa, possibilitando o uso de instrumentos com menor tensão de operação (Chapman, 2013).

Figura 2: Conexão dos instrumentos convencionais ao transformador para ensaio de circuito aberto.

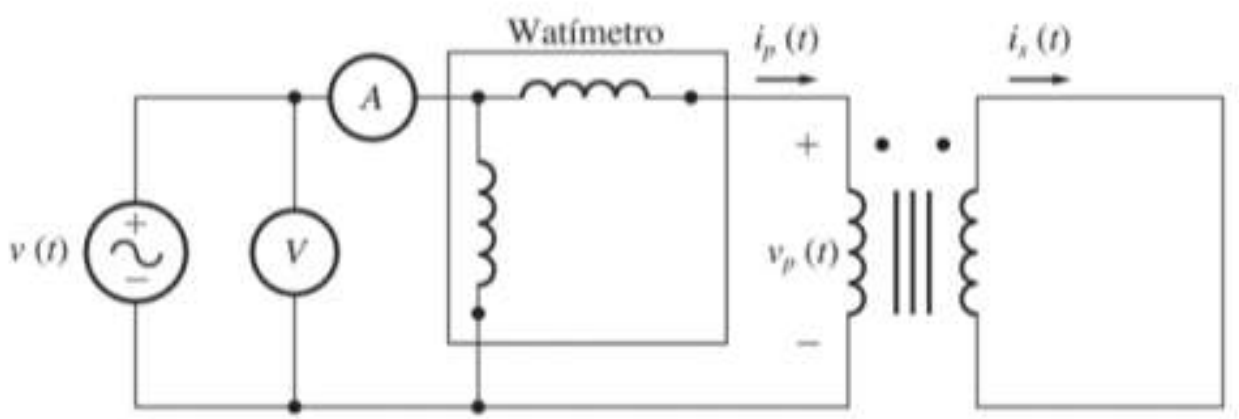

Transformador

Fonte: Chapman (2013).

Para realização do ensaio de curto-circuito é necessário fornecer a corrente nominal a um dos enrolamentos, isso é feito curto-circuitando um dos seus lados e aplicando tensão até surgir a corrente nominal. Considerando que a impedância de 
magnetização possui um valor muito maior que a impedância série, entende-se que grande parte da corrente flui pelas resistências de enrolamentos e reatâncias de dispersão (Toro, 2016). Dessa forma, a Figura 3 mostra o arranjo para o ensaio de curto-circuito.

Figura 3: Conexão dos instrumentos convencionais ao transformador para ensaio de curto-circuito.

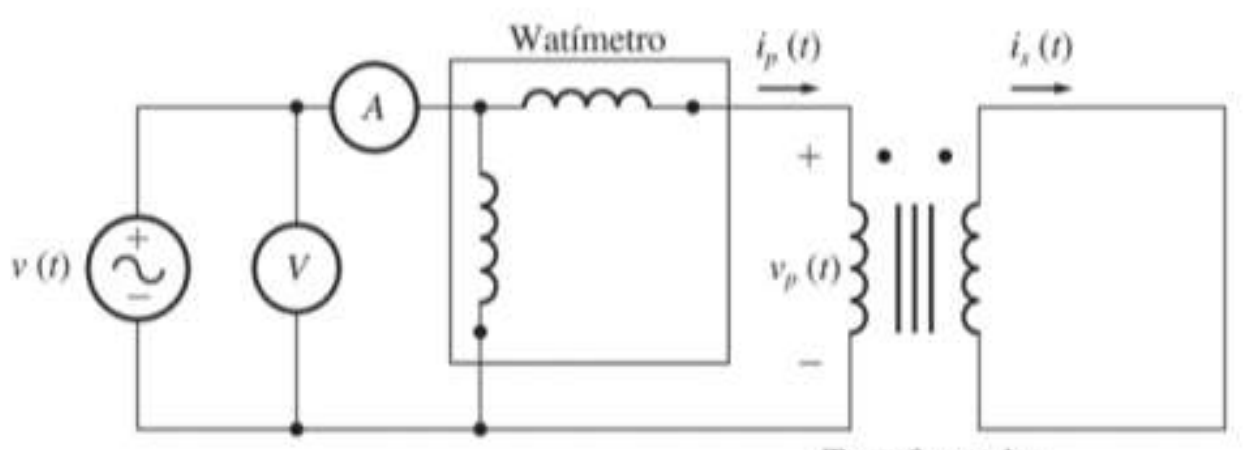

Transformador

Fonte: Chapman (2013).

A tensão aplicada para surgimento da corrente nominal está entre $10 \%$ a $15 \%$ da tensão nominal. Sendo aconselhável realizar os procedimentos do ensaio de curto-circuito no lado de alta (Jordão, 2002).

\subsection{Instrumentação Virtual e LabVIEW}

O desenvolvimento de tecnologias computacionais vem acontecendo mais intensamente nas últimas décadas, os computadores pessoais apresentam processadores mais rápidos e sistemas operacionais mais intuitivos. A internet também vem sendo aprimorada, permitindo acessos mais velozes e com maior banda de transferência. A instrumentação virtual se beneficia desses avanços para obter maiores taxas de amostragem e processamento dos dados (Cao \& Chen, 2014).

Uma excelente aplicação de instrumentação virtual seria na aquisição de grandezas elétricas em laboratórios, assim, os custos com a obtenção de aparelhos de medição e controle necessários para a montagem das bancadas experimentais seriam reduzidos. Surge-se também a possibilidade de inserir outras funções aos medidores virtuais, através do software, sem a necessidade de alterar o hardware utilizado na montagem experimental, fazendo com que as medições sejam mais dinâmicas e multifuncionais (Lopes, 2007).

Para criação é necessário a utilização de alguma linguagem de programação que possibilite e torne esse processo o mais simples possível. Entre várias linguagens existentes, a linguagem G (linguagem gráfica) é a mais indicada para esse tipo de aplicação, já que, ao comparar-se com linguagens textuais, ela traz métodos de aprendizado mais rápidos, e ferramentas destinadas à aplicação de instrumentos virtuais ( $\mathrm{Si}, 2014)$.

O LabVIEW é uma linguagem de programação gráfica, capaz de fornecer a criação de testes, medições, aquisição de dados, controle de instrumentos, armazenamento de dados, análise de dados e geração de relatórios. A National Instruments, desenvolvedora do LabVIEW, foi fundada em 1986 com o intuito de criar um ambiente de programação voltado para engenheiros e cientistas, e atualmente está inserida nas mais diversas áreas de tecnologia (Emami \& Yazdi, 2009; Higa; Tawy; \& Lord, 2003).

Os softwares criados na linguagem de programação gráfica LabVIEW são chamados de VI's (virtual instruments), remetendo a sua motivação de criação, que era desenvolver sistemas e programas que simulem instrumentos convencionais de forma virtual. O LabVIEW comunica-se com uma grande quantidade de hardwares, sejam eles da própria National Instruments ou de terceiros, em ambos os casos, existem funções e blocos de código prontos que tornam o processo de conectar dispositivos 
bem mais rápidas e simples. Ele também permite a interação com outras linguagens de programação, podendo traduzi-las em LabVIEW ou o inverso (Agraz \& Pozos, 2013).

\subsection{Instrumentação Virtual aplicada a ensaios automatizados}

Os procedimentos estabelecidos nesse trabalho para ensaios de curto-circuito e circuito aberto em transformadores monofásicos seguem as mesmas etapas descritas na Seção 2.2. Porém, apesar da semelhança nos processos, a diferença é vista na aplicação dos instrumentos de medição e controle, ocorrendo na substituição da operação manual de quatro diferentes instrumentos convencionais (fonte de tensão alternada, amperímetro, voltímetro e wattímetro) por um único instrumento virtual operado de modo automático.

A Figura 4 permite entender a dinâmica do sistema de ensaios automatizados desenvolvido através de um diagrama de blocos simplificado. Dentre os materiais a serem utilizados no desenvolvimento da bancada ressalta-se a fonte AC Keysight, sensores de tensão, sensores de corrente, o dispositivo de aquisição (DAQ) USB-6002, relés, contatores e o software LabVIEW.

Figura 4: Diagrama de blocos que mostra a dinâmica do sistema de ensaios automatizados de transformadores.

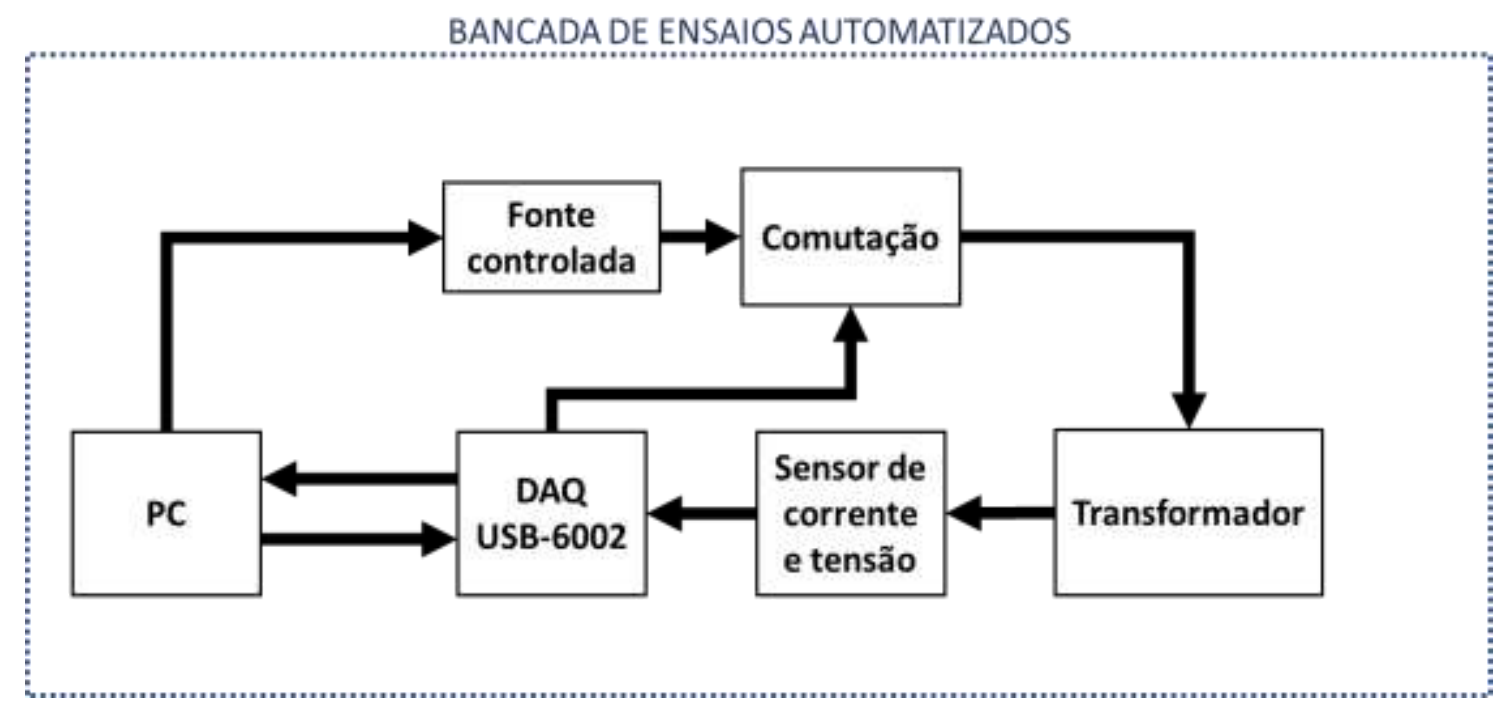

Fonte: Autores.

Em síntese, o diagrama de blocos mostra que, através de um sistema de aquisição de dados, os sensores de tensão e corrente captam os sinais do transformador e os enviam ao DAQ USB-6002, que os condicionam e convertem para sinal digital. Esses sinais são transmitidos para o computador que tem instalado o software LabVIEW por meio de uma conexão USB. Com a leitura das formas de onda da tensão e corrente, é possível calcular a potência ativa, obtendo assim, o circuito equivalente do transformador, as perdas no cobre e as perdas por magnetização.

Para que ambos ensaios possam ser realizados é necessário alternar a disposição de conexão dos sensores e da fonte de alimentação ao transformador, de acordo com o tipo de ensaio selecionado. Além disso, o LabVIEW permite controlar pelo computador a tensão de saída da fonte para as amplitudes e valores RMS desejados em cada um dos ensaios.

\section{Resultados e Discussão}

\subsection{Aspectos construtivos da bancada de ensaio}

Visando a conexão dos transformadores monofásicos de baixa tensão ao sistema automatizado de ensaios foi elaborada a bancada mostrada na Figura 5, a qual permitiu de modo simples a preparação e acoplamento de todos os elementos 
necessários a execução dos ensaios de circuito aberto e curto-circuito. Os elementos integrantes da bancada são: fonte controlada de alimentação CA, suporte para alocação do transformador e o quadro de comando.

Figura 5: Bancada de ensaio automatizado montada.

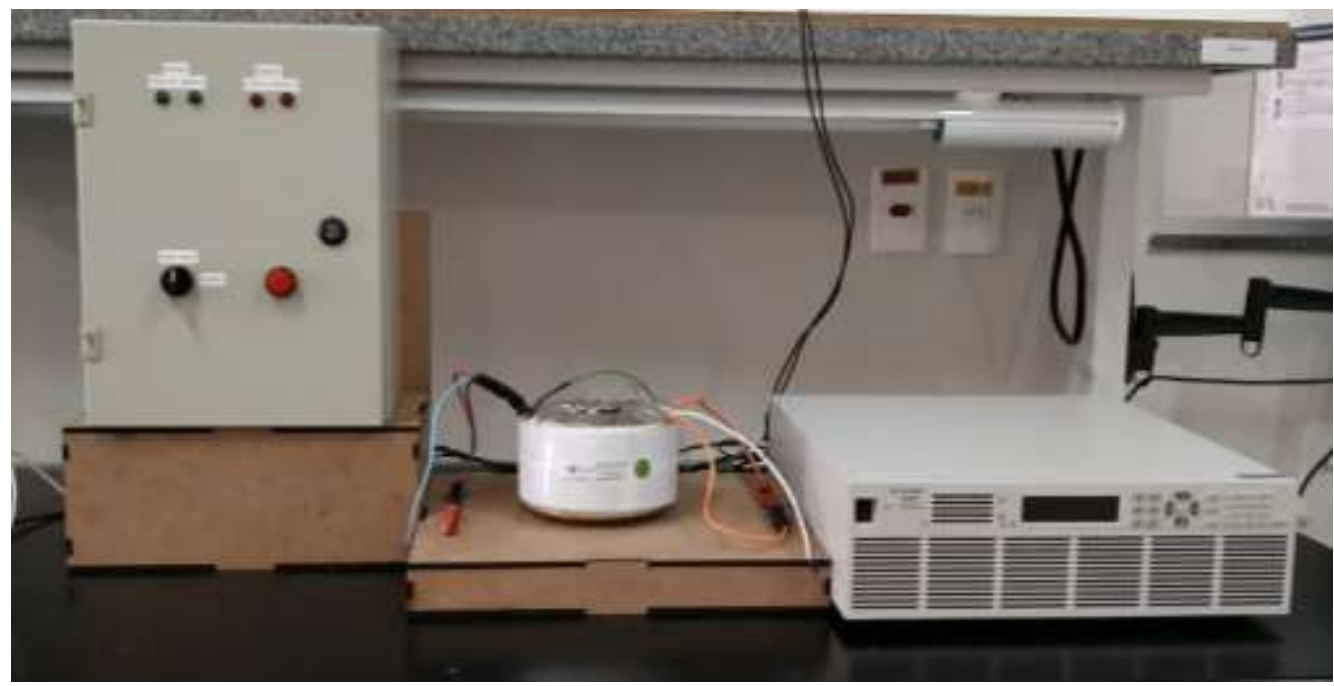

Fonte: Autores.

O sistema foi desenvolvido com base na fonte de tensão alternada Keysight AC6803A, com tensão de saída máxima de 270 Vrms, com corrente nominal de 20 Arms e com potência nominal de 2000 VA. Outra característica importante é que, através do LabVIEW, ela tem a capacidade de controle em tempo real da tensão de saída e suas características, pois possui drivers disponibilizados gratuitamente que permitem a comunicação com o computador. É importante salientar que a fonte foi o fator limitante quanto a potência, tensão e corrente máxima dos transformadores ensaiados.

O quadro de comando é também uma parte fundamental do sistema de ensaios automatizados, visto que, internamente, ele abriga os circuitos de proteção, de comutação e de aquisição de sinais. A Figura 6 mostra mais detalhadamente os componentes responsáveis pela proteção e comutação entre os ensaios.

Figura 6: Disjuntores e contatores presentes no quadro de comando do sistema.

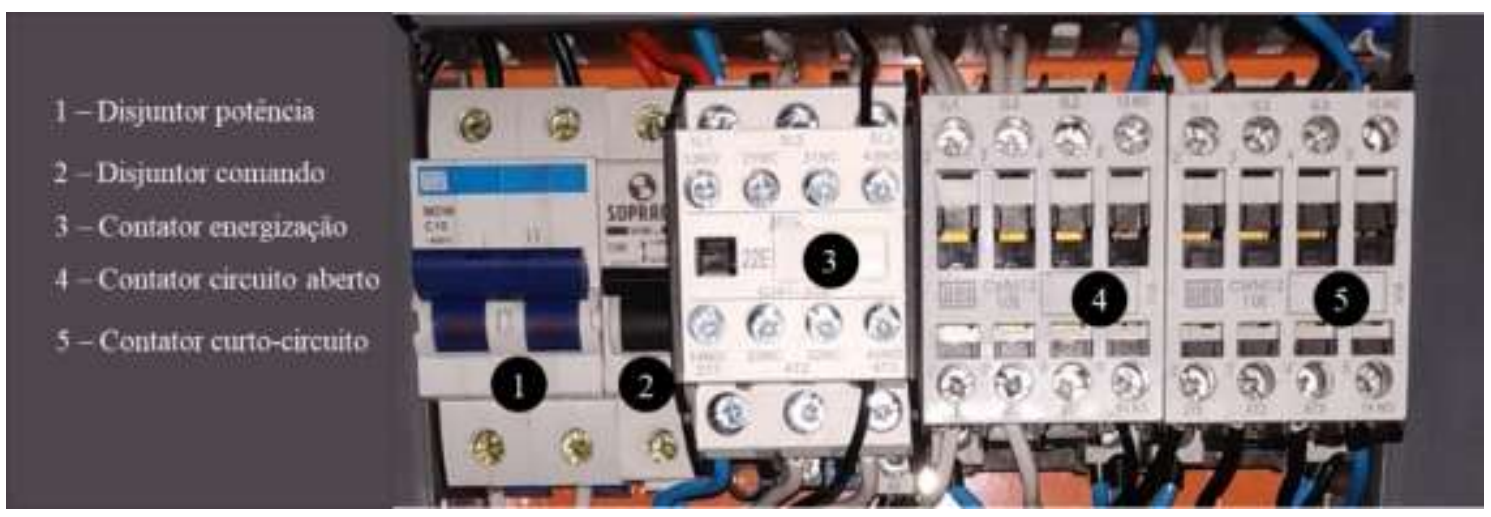

Fonte: Autores.

Os disjuntores são responsáveis por proteger o sistema e o transformador ensaiado contra sobrecarga e surtos de corrente. Os contatores são responsáveis pelas conexões em diferentes configurações durante os ensaios, um deles destinado ao ensaio de curto-circuito e o outro destinado ao ensaio de circuito aberto. O acionamento dos contatores é executado usando a 
saída analógica do DAQ-USB6002 e para isso, foi desenvolvido uma placa de circuito impresso (Figura 7) com os dois relés e seu circuito para acionamento. Um dos relés aciona o contator do ensaio de curto-circuito e o outro relé aciona o contator do ensaio de circuito aberto.

Figura 7: Placa de circuito impresso para aquisição de sinais e atuação.

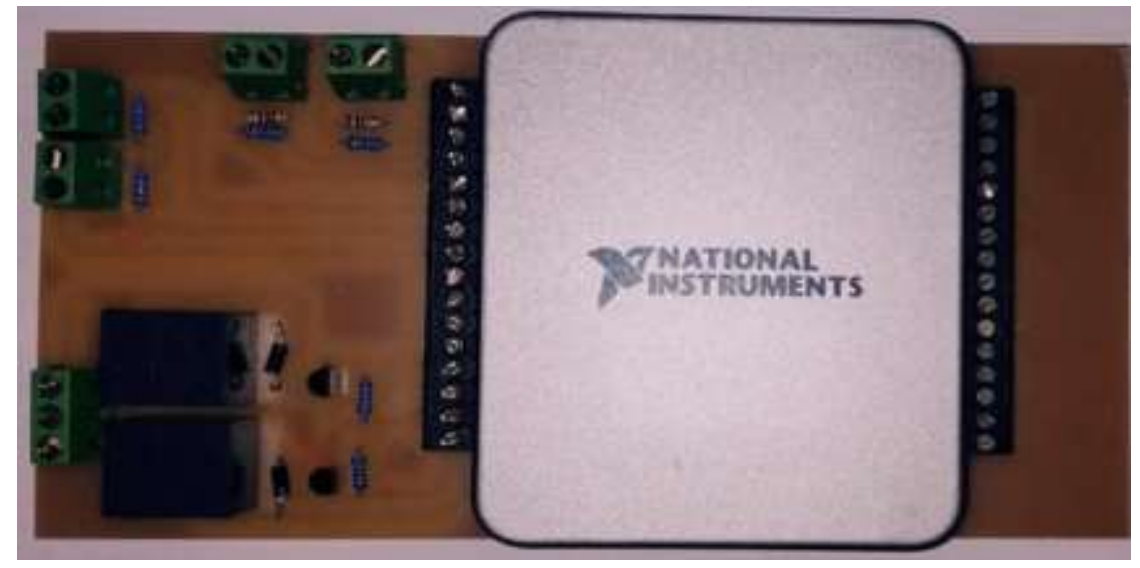

Fonte: Autores.

A Figura 7 ainda revela: o circuito para conexão do sensor de corrente, o divisor resistivo para medição de tensão e o NI USB-6002. O sensor de corrente utilizado é um transformador de corrente que mede até $100 \mathrm{~A}$, com relação de transformação de 2000 vezes. O divisor de tensão foi projetado para uma tensão máxima de $300 \mathrm{~V}$ e com uma tensão de saída com valor próximo a $5 \mathrm{~V}$ pico a pico.

A placa DAQ NI USB-6002 da National Instruments possui conexão por barramento USB, possui 8 entradas analógicas de 16 bits de resolução e taxa de amostragem total de $50 \mathrm{Ksamples/s,} \mathrm{dessas,} \mathrm{é} \mathrm{possível} \mathrm{trabalhar} \mathrm{de} \mathrm{modo} \mathrm{referenciado}$ e de modo diferencial. Há também 2 saídas analógicas com 16 bits de resolução e taxa de atualização de $5 \mathrm{KHz}, 13$ canais digitais que podem ser configurados como entrada ou saída, e 1 contador de 32 bits de $5 \mathrm{MHz}$ (Filho et al., 2018; Waterkemper et al., 2018).

\subsection{Software desenvolvido em LabVIEW}

A criação do software que comanda todo o hardware, adquire sinais dos sensores e comunica com a fonte controlada foi implementada da linguagem de programação gráfica LabVIEW. A interface gráfica foi projetada de modo que permitisse a interação do usuário com o programa de maneira amigável e intuitiva.

Como pode ser verificado na Figura 8, a interface gráfica é composta pelo menu lateral esquerdo e pela janela principal. Clicando em algum dos botões no menu, a janela principal altera-se, sendo assim, o operador do ensaio pode selecionar opções de começar um novo ensaio, de ver o último relatório gerado, de alterar os valores de calibração dos sensores e de mudar as configurações relativas à fonte de tensão controlada e o DAQ. 
Figura 8: Tela para configuração de um novo ensaio.

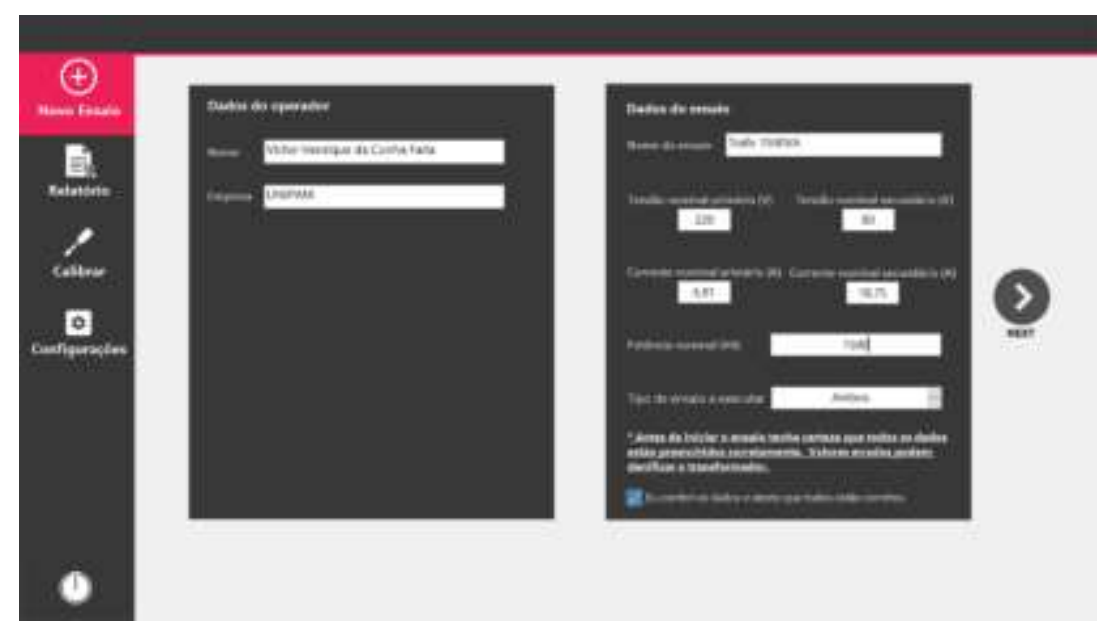

Fonte: Autores.

Ao selecionar o botão "Novo ensaio" no menu lateral esquerdo, o usuário é direcionado a tela referente a Figura 8. Está é a tela em que o usuário deverá preencher os dados relativos ao operador que executará o ensaio e os dados relativos ao transformador que será ensaiado. Sendo a etapa de preenchimento de dados relativos ao transformador uma das mais importantes do sistema, pois é o local em que são fornecidas as informações primordiais para realização dos ensaios, assim como: tensões e correntes de ambos os enrolamentos e a potência nominal.

Durante o andamento dos ensaios, alguns dados são importantes para que o operador entenda o que está acontecendo e possa intervir através do comutador no quadro de comando, caso algo saia diferente dos conformes. A Figura 9 exibe a janela em que é detalhado os processos executados no decorrer dos ensaios.

Figura 9: Tela de acompanhamento dos processos durante o ensaio.

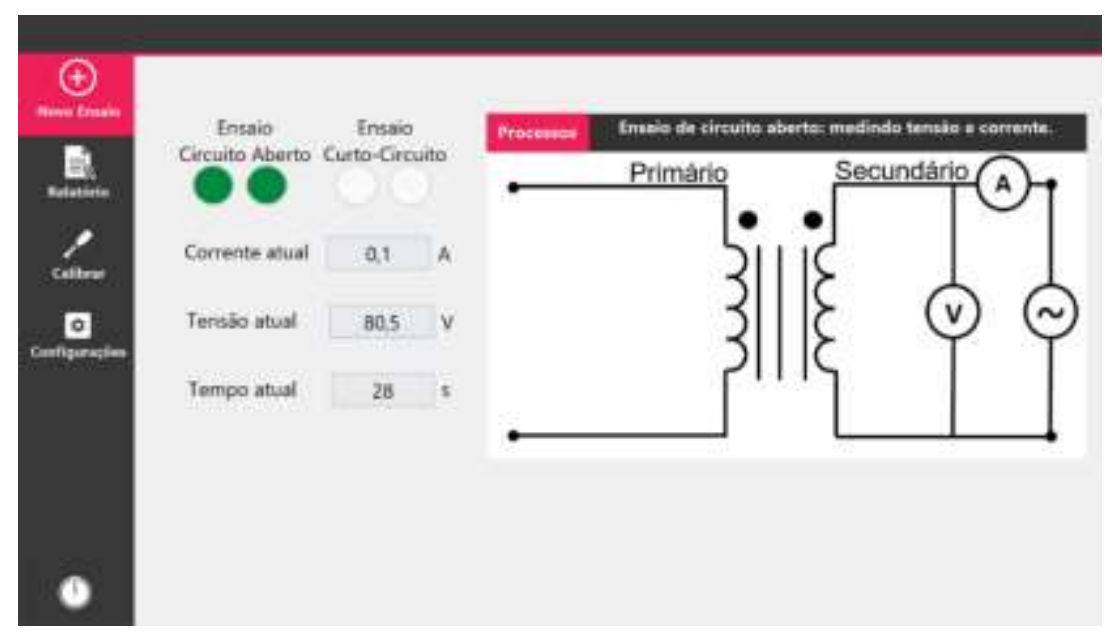

Fonte: Autores.

Nessa janela existem sinalizadores para mostrar de forma clara qual ensaio está ocorrendo, indicadores numéricos que permitem visualizar a corrente e tensão fornecidas ao transformador e o tempo atual demandado. Também, ao lado direito da janela existe o painel de informação, este é responsável por mostrar de forma textual e gráfica qual o processo específico está sendo realizado (zerando tensão da fonte, aumentando tensão da fonte, realizando medições, calculando). Ao fim dos ensaios, o sistema utiliza as informações coletadas de tensão e corrente para calcular os dados propostos por este projeto. 
Com todos os valores obtidos, o software disponibiliza a opção de visualizar os resultados gerados. A Figura 10 exemplifica a tela de relatórios do ensaio de circuito aberto, apesar de ser bem semelhante à de relatório de curto-circuito, elas apresentam diferenças entre si.

No relatório de circuito aberto exibe-se os valores de tensão RMS, corrente Rms, Rmag, Xmag, potência ativa (perdas por magnetização), potência reativa, potência aparente, fator de potência, defasagem angular e mostra graficamente as ondas de tensão e corrente. Por outro lado, o relatório de ensaio de curto-circuito tem os valores de tensão RMS, corrente RMS, Rcc, Xcc, potência ativa (perdas de curto-circuito), potência reativa, potência aparente, fator de potência, defasagem angular e mostra graficamente as ondas de tensão e corrente.

Figura 10: Tela de relatórios dos ensaios de circuito aberto.

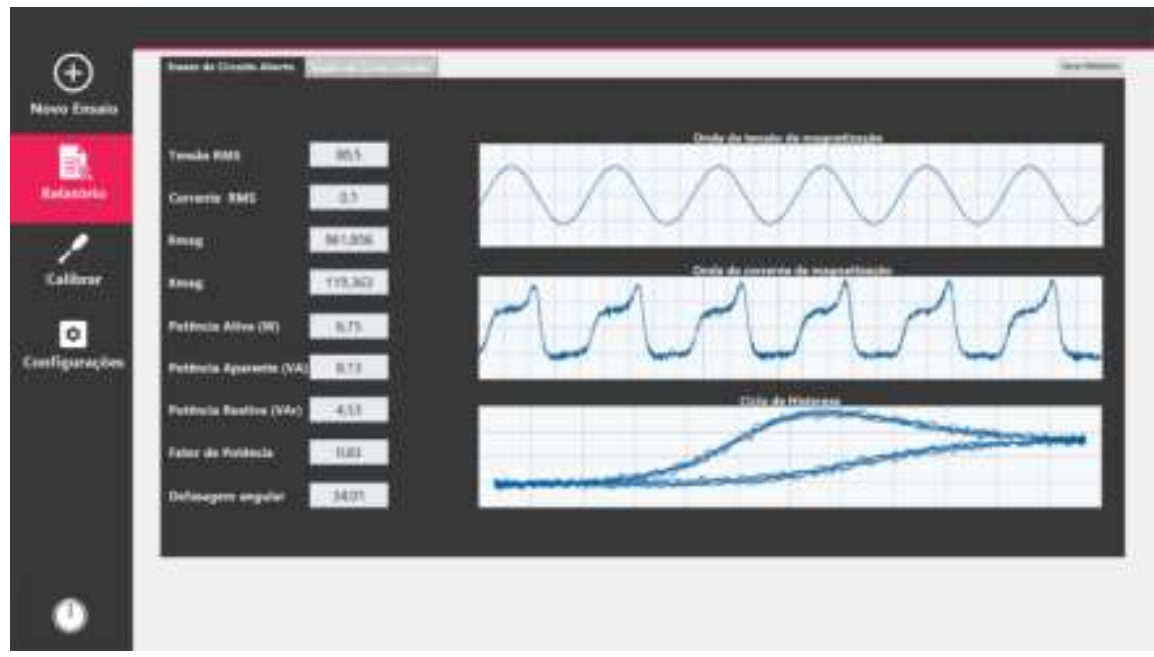

Fonte: Autores.

Outro recurso importante é o de criar relatórios em documentos de texto, para tal, basta pressionar o botão “Imprimir Relatório", no canto inferior direito, verificado na Figura 10. Logo após pressionado, o arquivo em Microsoft Word surgirá automaticamente, já com os valores adquiridos durante os ensaios. Dessa forma, para que esse recurso funcione, é preciso que o Microsoft Word também esteja instalado no dispositivo, juntamente com o software.

Figura 11: Funções que compõem o ensaio de circuito aberto.

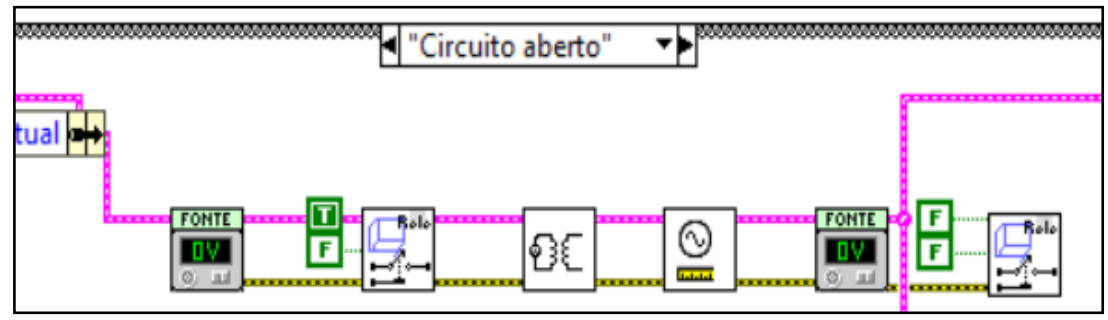

Fonte: Autores.

A Figura 11 permite visualizar seis funções referentes ao ensaio de circuito aberto. Primeiro, de acordo com o fluxo de dados, verifica-se a tensão atual no sistema através dos sensores de tensão, e caso seja diferente de $0 \mathrm{~V}$, a fonte será zerada gradativamente. Em segundo lugar, certificado que a fonte está zerada, aciona-se o relé do ensaio de circuito aberto, conectando a fonte ao secundário do transformador e abrindo o primário. 
Em terceiro, com as conexões prontas, é aumentado a tensão da fonte até o valor nominal fornecido pelo usuário, acrescentando-se gradualmente enquanto é medido o valor de tensão pelo sensor. Ao chegar na tensão buscada, passa-se para o quarto ponto: as medições. Nesse passo, são efetuadas dez medições de tensão e corrente, espaçadas um segundo entre elas, e então, faz-se a média destes valores.

Com as medições realizadas, obtém-se a tensão e corrente RMS e as ondas de tensão e corrente, o quais já são suficientes para calcular os elementos do circuito equivalente do transformador. O quinto ponto é zerar novamente a fonte para que o ensaio seja encerrado e seja possível abrir o contator referente ao ensaio de circuito aberto.

A programação chave do ensaio de curto-circuito segue a mesma lógica da programação antemão citada, do ensaio de circuito aberto. Vê-se as funções que a integram na Figura 12.

Figura 12: Funções que compõem o ensaio de curto-circuito.

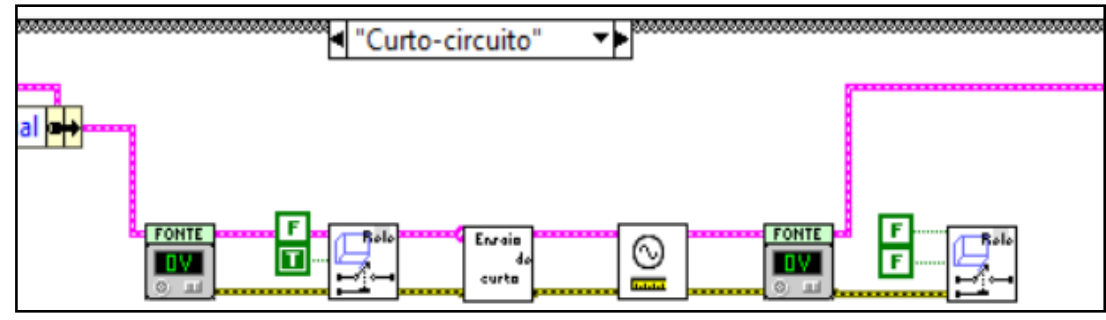

Fonte: Autores.

Em síntese, o código da Figura 12 realiza o seguinte: diminui-se a tensão a 0 volts, aciona-se o relé responsável por conectar a fonte ao primário do transformador e curto-circuitar o secundário, nesse momento, é aumentado a tensão lentamente até a corrente nominal, fornecida pelo usuário, a ser lida pelo sensor de corrente. É medido tensão e corrente, e por fim, zera-se novamente a fonte e desatraca os contatores. Em ambos os ensaios, após a etapa de medição, são realizados os cálculos e exibido os resultados nos relatórios.

Em vista de averiguar a eficiência do sistema (bancada, interface de usuário e o código) foram realizados alguns testes, comparando os ensaios do sistema com o método convencional. Os resultados dos testes e detalhes de execução estão dispostos na seção conseguinte.

\subsection{Testes para validação da bancada}

O sistema automatizado de ensaio de curto-circuito e circuito aberto foi testado utilizando dois transformadores de baixa tensão, do laboratório de Conversão de Energia do UNIPAM, com aspectos construtivos, potência nominal e tensão nominal diferentes, de modo a diversificar os testes e atestar a eficiência do sistema automatizado de ensaios.

Figura 13: Transformadores ensaiados durante os testes.

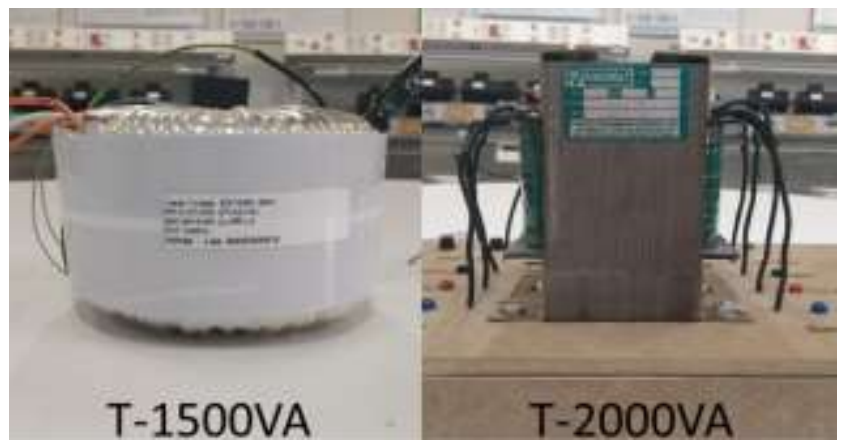

Fonte: Autores. 
Dentre os transformadores, um tem o núcleo ferromagnético toroidal (T-1500VA) e é dedicado a conversão do nível de tensão em aplicações nos laboratórios do UNIPAM, e o outro, T-2000VA, tem o núcleo em formato convencional e é empregado em fins didáticos no ensino de Engenharia Elétrica. Aspectos importantes quanto aos transformadores da Figura 13 (modelo, a potência nominal, a frequência de operação e a quantidade de derivações (taps) nos enrolamentos), estão relacionados na Tabela 1.

Tabela 1: Características dos transformadores empregados nos ensaios para teste do sistema.

\begin{tabular}{cccccc}
\hline Transformador & Fases & Modelo & $\begin{array}{c}\text { Potência nominal } \\
(\text { VA) }\end{array}$ & $\begin{array}{c}\text { Taps } \\
\text { Primário (V) }\end{array}$ & $\begin{array}{c}\text { Taps } \\
\text { Secundário (V) }\end{array}$ \\
\hline T-1500VA & 1 & TP15936 & 1500 & $0-127-220$ & $40-0-40$ \\
T-2000VA & 1 & TMF0117 & 2000 & $127-220-380$ & $127-220-380$ \\
\hline
\end{tabular}

Fonte: Autores.

Consoante a Tabela 1 , todos os transformadores são monofásicos e tem potência elétrica nominal menor que a potência nominal da fonte de tensão. Além disso, eles apresentam frequência nominal de $60 \mathrm{~Hz}$ e tem três derivações cada, todas elas com tensão nominal menor que $270 \mathrm{~V}$. Tais fatores os qualificam para serem ensaiados pelo sistema desenvolvido nesse projeto, pois têm parâmetros elétricos condizentes com a fonte de alimentação.

Ao longo dos testes foram definidos quais taps de cada transformador seriam utilizados, preenchendo então, os dados do ensaio na tela de configuração de acordo com a Tabela 2.

Tabela 2: Caraterísticas dos transformadores fornecidas ao sistema durante os ensaios.

\begin{tabular}{cccccc}
\hline Transformador & $\begin{array}{c}\text { Potência } \\
\text { Nominal }\end{array}$ & $\begin{array}{c}\text { Tensão } \\
\text { nominal } \\
\text { primário }\end{array}$ & $\begin{array}{c}\text { Corrente } \\
\text { nominal } \\
\text { primário }\end{array}$ & $\begin{array}{c}\text { Tensão } \\
\text { nominal } \\
\text { secundário }\end{array}$ & $\begin{array}{c}\text { Corrente } \\
\text { nominal } \\
\text { secundário }\end{array}$ \\
\hline T-1500VA & $1500 \mathrm{VA}$ & $220 \mathrm{~V}$ & $6,81 \mathrm{~A}$ & $80 \mathrm{~V}$ & $18,75 \mathrm{~A}$ \\
T-2000VA & $2000 \mathrm{VA}$ & $220 \mathrm{~V}$ & $9,09 \mathrm{~A}$ & $127 \mathrm{~V}$ & $15,74 \mathrm{~A}$ \\
\hline
\end{tabular}

Fonte: Autores.

Em cada um dos transformadores foi executado cinco ensaios através do sistema desenvolvido, aguardando 10 minutos entre cada ensaio. Em paralelo ao sistema de ensaios automatizados, conectou-se o osciloscópio mostrado na Figura 27 , buscando averiguar os valores encontrados nos ensaios e certificar que eles estavam corretos. 
Figura 14: Sistema automatizado de ensaios com osciloscópio conectado para averiguar a fidelidade das medições.

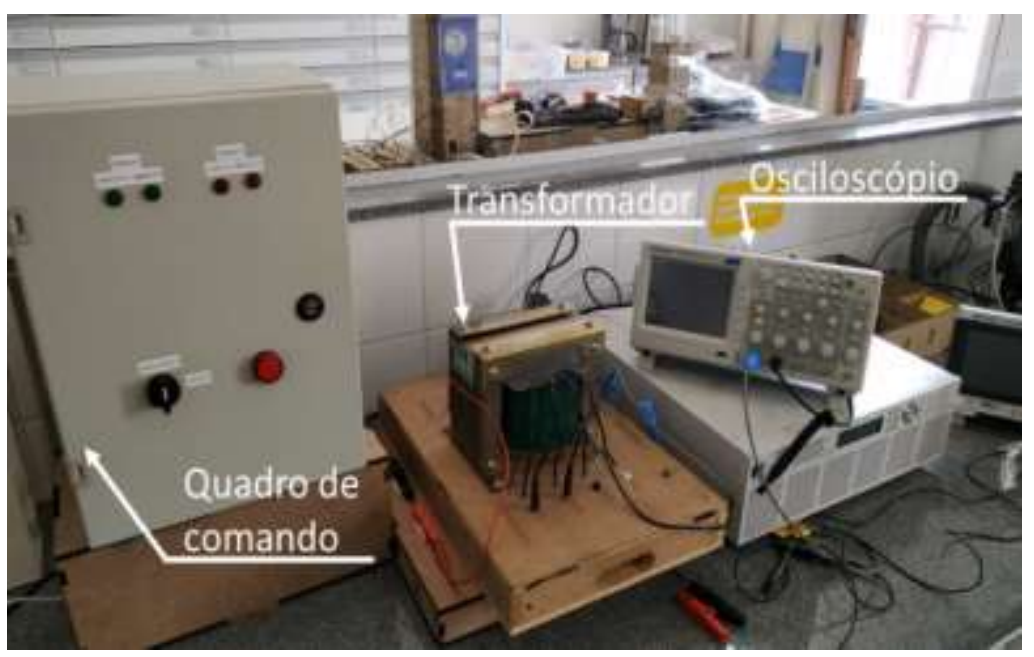

Fonte: Autores.

O osciloscópio utilizado foi o TDS2014C da Tektronix, em conjunto com a ponteira de tensão TekP5122 e o alicate amperímetro TekA622. Deste modo, para todo ensaio do sistema automatizado, registrou-se os valores correspondentes de tensão RMS, corrente RMS e potência ativa, aferidos pelo osciloscópio. Por fim, com os dados obtidos do osciloscópio, calculou-se os valores de impedância (resistência de magnetização, reatância de magnetização, resistência do enrolamento e reatância de dispersão) dos ensaios usando uma tabela desenvolvida no Excel.

A Tabela 3 e a Tabela 4 reúnem a média e o erro relativo entre as medições das grandezas obtidas pelo sistema automatizado de ensaio e pelo osciloscópio, englobando o ensaio de circuito aberto e de curto-circuito do T-1500VA.

Tabela 3: Resultado dos ensaios de circuito aberto no T-1500VA.

\begin{tabular}{cccccc}
\hline $\begin{array}{c}\text { Ensaio de Circuito } \\
\text { Aberto }\end{array}$ & $\begin{array}{c}\text { Tensão } \\
\text { RMS }\end{array}$ & Corrente RMS & $\begin{array}{c}\text { Perda } \\
\text { magnetização }\end{array}$ & Rm $_{\text {LA }}$ & $\mathbf{X m}_{\text {LA }}$ \\
\hline $\begin{array}{c}\text { Média das medições } \\
\text { sistema }\end{array}$ & $80,024 \mathrm{~V}$ & $0,133 \mathrm{~A}$ & $8,240 \mathrm{~W}$ & $5,182 \mathrm{~K} \Omega$ & $6,324 \mathrm{~K} \Omega$ \\
$\begin{array}{c}\text { Média das medições } \\
\text { Osciloscópio }\end{array}$ & $80,312 \mathrm{~V}$ & $0,136 \mathrm{~A}$ & $8,020 \mathrm{~W}$ & $5,369 \mathrm{~K} \Omega$ & $5,934 \mathrm{~K} \Omega$ \\
Erro relativo & $0,36 \%$ & $1,95 \%$ & $-2,67 \%$ & $3,61 \%$ & $-6,17 \%$ \\
\hline
\end{tabular}

Fonte: Autores. 
Tabela 4: Resultado dos ensaios de curto-circuito no T-1500VA.

\begin{tabular}{cccccc}
\hline $\begin{array}{c}\text { Ensaio de } \\
\text { Curto-circuito }\end{array}$ & $\begin{array}{c}\text { Tensão } \\
\text { RMS }\end{array}$ & Corrente RMS & $\begin{array}{c}\text { Perda no } \\
\text { enrolamento }\end{array}$ & Rcc $_{\text {LA }}$ & Xcc $_{\text {LA }}$ \\
\hline $\begin{array}{c}\text { Média das medições } \\
\text { sistema }\end{array}$ & $10,226 \mathrm{~V}$ & $6,865 \mathrm{~A}$ & $67,281 \mathrm{~W}$ & $1,428 \Omega$ & $0,454 \Omega$ \\
$\begin{array}{c}\text { Média das medições } \\
\text { Osciloscópio }\end{array}$ & $10,390 \mathrm{~V}$ & $6,868 \mathrm{~A}$ & $67,000 \mathrm{~W}$ & $1,420 \Omega$ & $0,520 \Omega$ \\
Erro relativo & $1,61 \%$ & $0,04 \%$ & $-0,42 \%$ & $-0,50 \%$ & $14,59 \%$ \\
\hline
\end{tabular}

Fonte: Autores.

As perdas por magnetização no T-1500VA foram de 8,240 W (2,67\% de ER), ao mesmo tempo que as perdas no cobre foram de $67,281 \mathrm{~W}(0,42 \%$ de ER). A tensão de ensaio de circuito aberto foi de 80,024 V e a de curto-circuito de 10,226 V, gerando correntes de 0,133 A e de 6,865 A, respectivamente. Com as impedâncias encontradas, referidas ao lado de alta, o circuito equivalente desse transformador é estabelecido por: 5,182 K $\Omega$ de resistência de magnetização, 6,324 K $\Omega$ de reatância de magnetização, 1,428 $\Omega$ de resistência do enrolamento e $0,454 \Omega$ de reatância de dispersão.

O erro relativo apresentado durante o ensaio de circuito aberto neste transformador, o T-1500VA, apresentou valores maiores que o do ensaio de circuito aberto do T-500VA em todas as grandezas, exceto a tensão RMS. Sendo que, o maior erro relativo deste ensaio aconteceu na reatância de magnetização, com 6,17\%. Já no ensaio de curto-circuito foi verificado um ER ainda maior, de 14,59\%, o qual ocorreu na reatância de dispersão, em erro absoluto, encontra-se um valor de $0,066 \Omega$.

A Tabela 5 e a Tabela 6 evidenciam os resultados do ensaio de circuito aberto e de curto-circuito do T-2000VA, a média e o erro relativo de medição dos valores.

Tabela 5: Resultado dos ensaios de circuito aberto no T-2000VA.

\begin{tabular}{cccccc}
\hline $\begin{array}{c}\text { Ensaio de Circuito } \\
\text { Aberto }\end{array}$ & $\begin{array}{c}\text { Tensão } \\
\text { RMS }\end{array}$ & Corrente RMS & $\begin{array}{c}\text { Perda } \\
\text { magnetização }\end{array}$ & $\mathbf{R m}_{\text {LA }}$ & $\mathbf{X m}_{\text {LA }}$ \\
\hline $\begin{array}{c}\text { Média das medições } \\
\text { sistema }\end{array}$ & $127,04 \mathrm{~V}$ & $2,370 \mathrm{~A}$ & $92,220 \mathrm{~W}$ & $0,547 \mathrm{~K} \Omega$ & $0,176 \mathrm{~K} \Omega$ \\
$\begin{array}{c}\text { Média das medições } \\
\text { Osciloscópio }\end{array}$ & $126,55 \mathrm{~V}$ & $2,317 \mathrm{~A}$ & $91,246 \mathrm{~W}$ & $0,548 \mathrm{~K} \Omega$ & $0,180 \mathrm{~K} \Omega$ \\
Erro relativo & $0,39 \%$ & $2,25 \%$ & $1,06 \%$ & $0,29 \%$ & $2,08 \%$ \\
\hline
\end{tabular}

Fonte: Autores. 
Research, Society and Development, v. 10, n. 16, e249101623890, 2021

(CC BY 4.0) | ISSN 2525-3409 | DOI: http://dx.doi.org/10.33448/rsd-v10i16.23890

Tabela 6: Resultado dos ensaios de curto-circuito no T-2000VA.

\begin{tabular}{|c|c|c|c|c|c|}
\hline $\begin{array}{c}\text { Ensaio de } \\
\text { Curto-circuito }\end{array}$ & $\begin{array}{l}\text { Tensão } \\
\text { RMS }\end{array}$ & Corrente RMS & $\begin{array}{c}\text { Perda no } \\
\text { enrolamento }\end{array}$ & $\operatorname{Rec}_{\mathrm{LA}}$ & $X \operatorname{Xcc}_{\mathrm{LA}}$ \\
\hline $\begin{array}{c}\text { Média das medições } \\
\text { sistema }\end{array}$ & $10,628 \mathrm{~V}$ & $9,131 \mathrm{~A}$ & $94,047 \mathrm{~W}$ & $1,125 \Omega$ & $0,285 \Omega$ \\
\hline $\begin{array}{l}\text { Média das medições } \\
\text { Osciloscópio }\end{array}$ & $10,612 \mathrm{~V}$ & $9,220 \mathrm{~A}$ & $95,196 \mathrm{~W}$ & $1,120 \Omega$ & $0,266 \Omega$ \\
\hline Erro relativo & $0,15 \%$ & $0,98 \%$ & $1,22 \%$ & $0,50 \%$ & $6,73 \%$ \\
\hline
\end{tabular}

Fonte: Autores.

O maior erro relativo nos ensaios do T-2000VA deu-se na reatância de dispersão (6,73\%), apresentando um valor absoluto de erro de 0,019 $\Omega$. A tensão nominal RMS em ambos os ensaios proporcionou ER baixos, inferiores a 1\%, diferente da corrente RMS, que apesar de baixo, foi de 2,25\% para corrente de magnetização.

Relativo as impedâncias, tem-se $0,547 \mathrm{~K} \Omega$ de resistência de magnetização e $0,176 \mathrm{~K} \Omega$ de reatância de magnetização vindas do ensaio de circuito aberto, e 1,125 $\Omega$ de resistência de enrolamento e 0,266 $\Omega$ de reatância de dispersão, do ensaio de curto-circuito. Como nas outras tabelas, as impedâncias estão referidas ao primário do transformador. As perdas constatadas no T-2000VA foram de 92,220 W na magnetização e de 94,047 W no enrolamento, com erros de 1,06\% e 1,22*\%, respectivamente.

Considerando os aspectos e os valores apresentados nos testes individuais, pode-se destacar alguns pontos a respeito de todos os testes. Primeiramente, dentre os dados de tensão adquiridos, o maior erro absoluto de tensão foi de aproximadamente $300 \mathrm{mV}$, permitindo concluir que a medição de tensão do sistema está dentro de um limite aceitável.

Apesar do sensor de corrente exprimir erros relativos maiores que o de tensão, notou-se que ao avaliar o valor absoluto, devido as baixas correntes de magnetização, esse erro foi de $89 \mathrm{~mA}$. Portanto, análogo a folha de dados do sensor, a variação na corrente encontrada está dentro dos parâmetros esperados, já que o sensor garante linearidade de 3\% somente em correntes maiores que $10 \mathrm{~A}$.

Consequentemente, por ser encontrada a partir do valor médio da multiplicação da onda de tensão pela de corrente, a potência em todos os casos mostrou-se quase semelhante a encontrada no osciloscópio, com ER máximo de 2,67\%. Além disso, como já citado anteriormente, devido ao fato de ser utilizado somente para o ensino, é coerente que o T-2000VA tenha tido perdas maiores que os outros transformadores, por haver menor preocupação em sua construção.

Outro fator que confirma a veracidade dos resultados, é a diferença de magnitude entre as impedâncias do ramo de magnetização e as impedâncias do cobre, sendo a impedância de magnetização bem maior em todos os testes. Também é notório que os maiores erros relativos estavam nas reatâncias de magnetização e dispersão, e isso é proporcional ao erro na potência e na corrente RMS.

\section{Conclusão}

O sistema de ensaios automatizados cumpriu com o papel desejado, apresentando em software e hardware, processos que diminuíssem gargalos oriundos dos ensaios manuais. Assim, o operador do ensaio necessita apenas realizar as ligações do primário e secundário do transformador e dar início ao teste pelo programa criado. Deste modo, os resultados são acessados sem nenhum processo manual de medição e conexão de instrumentos. 
O software é uma parte importante do objetivo proposto, entendendo que o mesmo é o contato do operador com o sistema. Seu desenvolvimento foi realizado de modo a oferecer uma interface amigável e intuitiva, permitindo ao operador iniciar um novo ensaio, acompanhar e estar ciente de todos os procedimentos realizados em tempo real, de forma simples.

Assim, olhando para os testes como um todo, os ensaios apresentaram resultados satisfatórios, evidenciando a eficiência do sistema em encontrar valores próximos aos reais. Porém, destaca-se a necessidade de um melhor condicionamento do sinal, entendendo como este pode interferir na confiabilidade dos resultados das medições.

Em trabalhos futuros recomenda-se maior preocupação com a etapa de condicionamento dos sinais de tensão e corrente, filtrando ruídos e amplificando os sinais dos sensores. Já que, uma pequena variação na medição, pode resultar em grandes erros nos cálculos das impedâncias do circuito equivalente do transformador ensaiado. Também é sugerido que seja adicionado ao sistema um canal de medições de temperatura, permitindo a análise da sua influência nos resultados.

\section{Referências}

Agraz, J. L., \& Pozos, RS (2013). Software de controle baseado em LabVIEW para projeto de instrumentação de sensor de força de dedo. 2013 IEEE AUTOTESTCON , 1-6.

Almeida, A. et al. (2010). Medidor virtual para ensaios em materiais magnéticos e transformadores. XVIII Congresso Brasileiro de Automática, 635-639.

Araujo, D. C. P. (2009). Estudo de metodologia e técnicas para execução de ensaios de resposta em frequência em transformadores de potência. 2009.220 f. Dissertação (Mestrado em Engenharia Elétrica). Universidade Federal de Minas Gerais, 1-5.

Baggini, A. (2016). Power Transformers - Introduction to measurement of losses. INTAS, 17.

Bandeira, M. I. da C. (2004). Automação do ensaio de saturação em transformadores de corrente utilizados em sistemas de transmissão de energia elétrica. 2004. 129 f. Dissertação (Mestrado em Metrologia). Universidade Federal de Santa Catarina, 13-15.

Barman, S. D. (2013). Modeling of high voltage power transformer winding for partial discharge test. 2013. 54 f. Dissertação (Mestrado em Engenharia Elétrica) - National Institute of Technology, 1-3.

Batista, T. C. et al. (2010). Sistema automático para ensaios de perdas em núcleos de transformadores monoáfsicos. IEEE. 1-5.

Cao, Y. \& Chen, H. (2014) Research on function and design of virtual instrument based on labVIEW technology. Journal of Chemical and Pharmaceutical Research, 1099-1104.

Chapman, S. J. (2013). Fundamentos de Máquinas Elétricas. McGraw-Hill, 65-152.

Emami, A., \& Yazdi, H. (2009). Designing and constructing transformer test system with aid of Labview. 2009 2nd International Conference on Adaptive Science \& Technology (ICAST), 169-174.

Filho, W. R. de O. et al. (2018). Desenvolvimento de um projeto de automação de uma máquina para ensaios de torção a frio e a quente. 23o CBECiMat, 69877000 .

Higa, M. L.; Taw, D. M.; \& Lord, S. M. (2002). An introduction to labview exercise for an electronics class. 32nd Annual Frontiers in Education. 13-16.

Jordão, R. G. (2002). Transformadores. Blucher, 1-35.

Kang, S., et al. (2014). Development of a teaching experiment platform for a signal generator based on LabVIEW. Harbin, 261-265.

Lopes, V. J. S. (2007). Instrumentação virtual aplicada ao ensino experimental de engenharia elétrica. 2007.108 f. Dissertação (Mestrado em Engenharia Elétrica). Escola Politécnica da Universidade de São Paulo, 19-33.

Maia, V. L. (2013). Projeto de um transformador planar para isolamento e chaveamento de tiristores. 2013.170 f. Dissertação (Mestrado em Engenharia Elétrica). Universidade Federal do Rio de Janeiro, 1-170.

Maria, G. G. B. (2015). Análise das respostas magnéticas exibidas pelo aço de grão não orientado (GNO) e grão orientado (GO) após carregamento cíclico por cisalhamento. 2015. 110 f. Dissertação (Mestrado em Engenharia de Materiais). Centro Federal De Educação Tecnológica De Minas Gerais, 1-54.

Marín, E. A. G. (2016). Modelagem e simulação de faltas internas entre espiras em transformadores de potência para avaliação de funções de proteção. 2016. 186 f. Dissertação (Mestrado em Engenharia Elétrica). Universidade Federal de Santa Catarina, 33-75.

Prado, D. C. S. do. (2012). Método de determinação de perdas por histerese nos materiais magnéticos submetidos a formas de onda de indução magnética com harmônicas. 2012. 125 f. Dissertação (Mestrado em Engenharia Elétrica). Universidade Federal de Santa Catarina, 33-53.

Si, H. (2014). Design of virtual function signal generator based on sound card. Applied Mechanics and Materials. v. 543-547, 850-853.

Toro, V. Del. (2016). Fundamentos de máquinas elétricas. Rio de Janeiro: LTC, 56-127. 
Research, Society and Development, v. 10, n. 16, e249101623890, 2021

(CC BY 4.0) | ISSN 2525-3409 | DOI: http://dx.doi.org/10.33448/rsd-v10i16.23890

Umans, S. D. (2014). Máquinas elétricas: de Fitzgerald e Kingsley. AMGH, 48-110.

Vicente, J. M. E. (2006). Uma Contribuição à Automação de Ensaios em Transformadores de Potência em Média Tensão. 2006.163 f. Tese (Doutorado em Engenharia Elétrica). Universidade Federal De Itajubá, 12-40.

Waterkemper, L. et al. (2018). Didactic system of supervision and data acquisition to evaluate the performance of hydraulic pumps. IEEE Latin America Transactions, 1113-1120. 